\title{
KLIPPEL-TRENAUNAY SYNDROME: A TROUBLESOME CONDITION
}

\author{
Serafino, S.*; Rodrigues, C*; Bernardino, M.**; Carvalho, S.** \\ Trainee; ${ }^{* *}$ Consultant at Instituto Português de Oncologia de Lisboa \\ Director of Anesthesiology Service: Dra Isabel Serralheiro
}

Klippel-Trenaunay syndrome (KTS) is a rare congenital disorder characterized by the triad of port-wine stains, varicose veins and bony/soft tissue hypertrophy involving an extremity. Besides the limbs, other areas may be affected, such as the spinal cord and neck/oropharynx, which pose important challenges to the anesthesiologist: airway management, controversy about neuraxial techniques and potential blood loss. Because of its rarity and the importance of the anesthetic management considerations it requires, we report a case at our institution.

- 47-year-old women, ASA III

- Past medical history: KTS, pulmonar thromboembolism, anticoagulation with rivaroxaban, hemithyroidectomy and neoadjuvant radiotherapy

- Scheduled for excision of popliteal sarcoma

- Preoperative evaluation: no signs of difficult airway, normal echocardiogram and blood analysis. Interruption of rivaroxaban two days before surgery

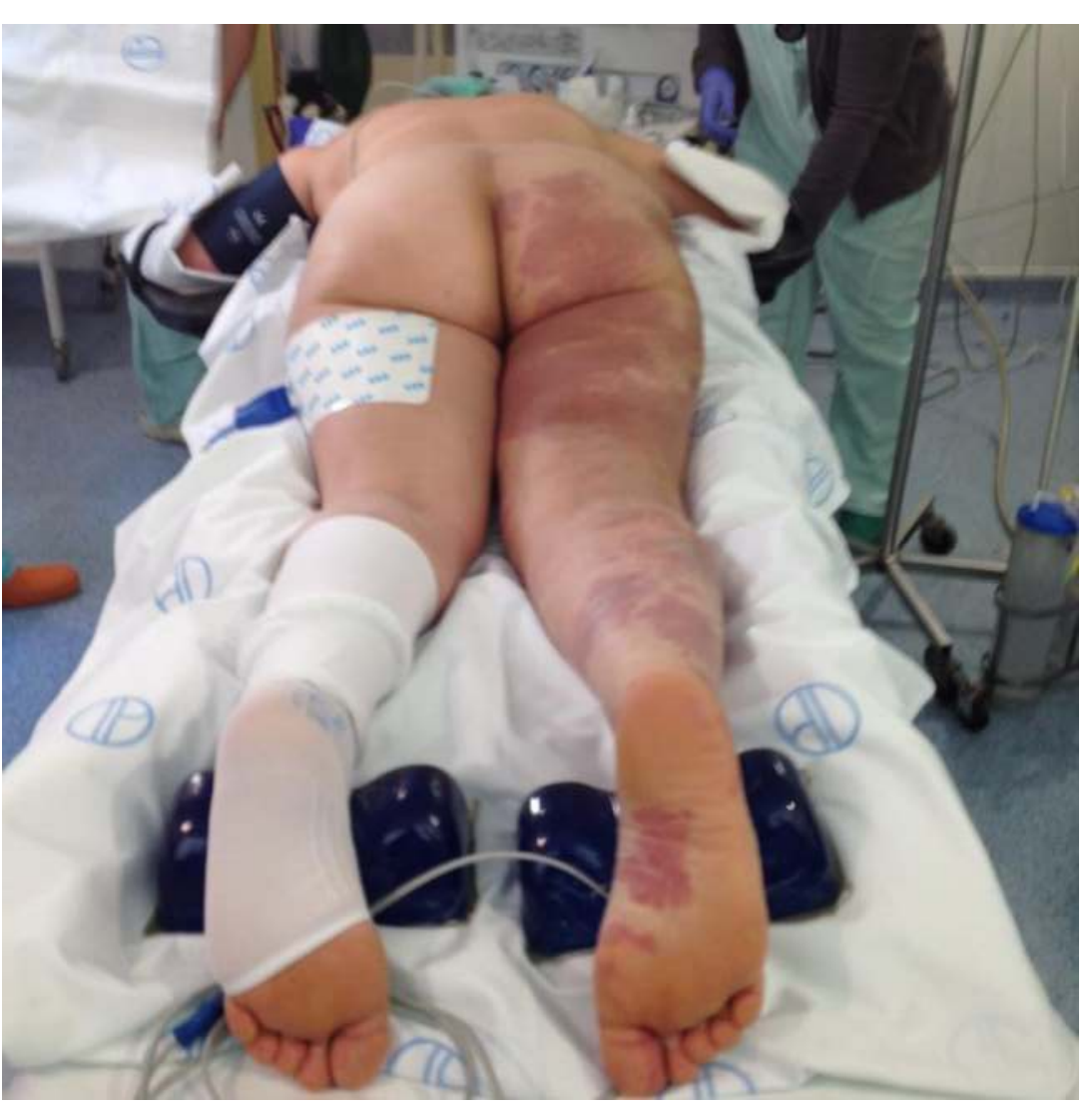

Fig. 1 - Port-wine stain typical of KTS

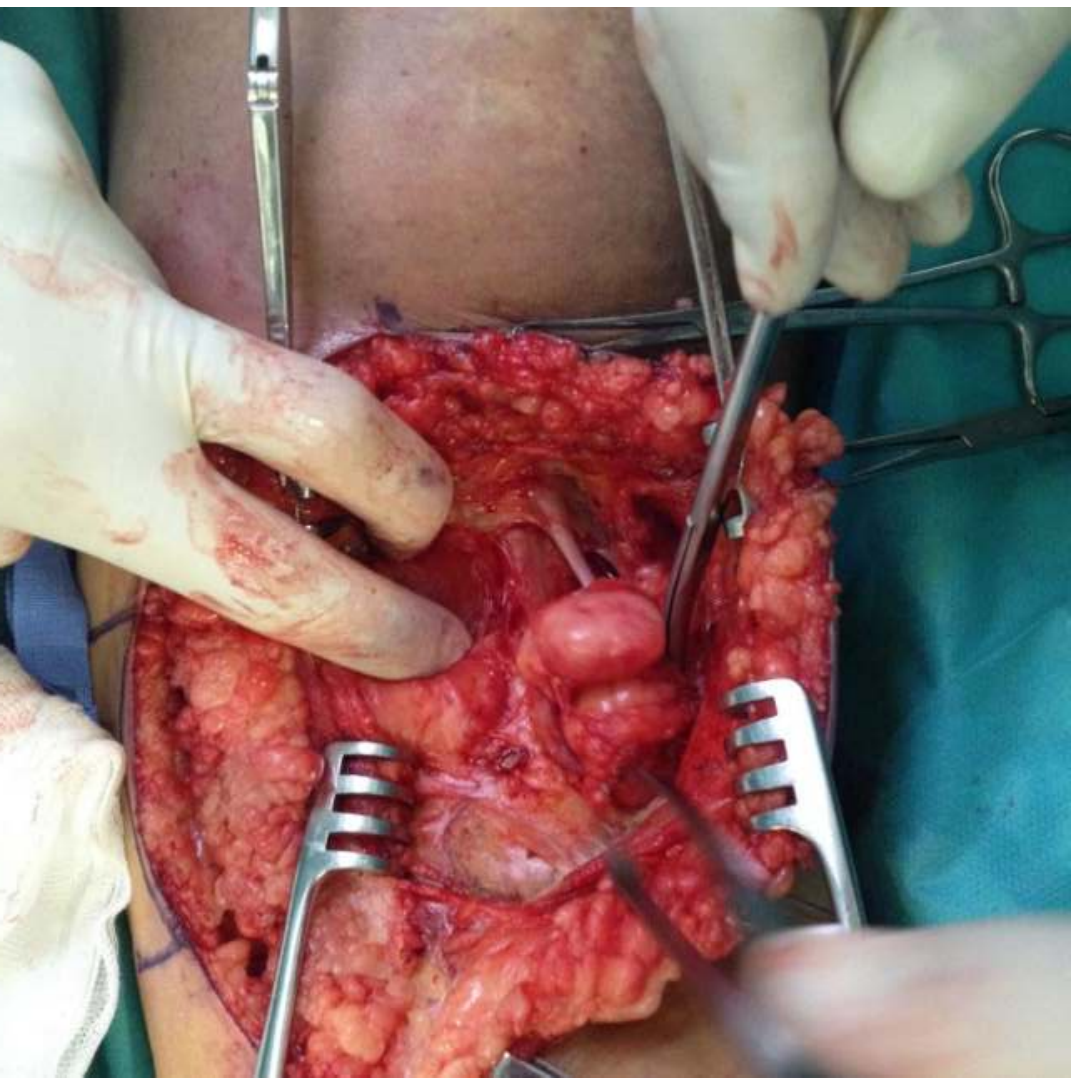

Fig. 2 - Excision of popliteal sarcoma

$\checkmark$ General anesthesia

$\checkmark$ Endotracheal intubation under direct laryngoscopy without difficulties

$\checkmark$ Hemodynamic stability and pain control was maintained and no significant blood loss was recorded

$\checkmark$ Neuraxial technique was NOT performed $\rightarrow$ No preoperative imaging studies were done to exclude neurovascular involvement

\section{Anesthetic management:}

\section{DISCIJSSION}

KTS incidence is low (1:27500) ${ }^{1}$ and its anesthetic management is still a matter of controversy. Despite previous reports of difficult airway management, a recent study ${ }^{1}$ didn't encounter significant airway difficulties, which was also the case of our patient. Blood loss is a potential problem, especially because these patients are frequently anticoagulated, but our patient didn't register significant haemorrhage. We didn't performed any neuraxial technique because no neuroimaging study was made before surgery. Still, this possibility can be considered in specific conditions, even if it is controversial ${ }^{2}$.

- $\quad$ Potential difficult airway is possible (even if rare)

- Massive blood loss should be anticipated

- $\quad$ Neuraxial techniques are controversial: may be considered only if vascular malformations were excluded by neuroimaging 